\title{
Energy-Efficient Clustering in IEEE 802.15.4 Wireless Sensor Networks
}

\author{
Hamidreza Tavakoli ${ }^{1}$, Jelena Mišić ${ }^{2}$, Majid Naderi ${ }^{1}$ and Vojislav B. Mišić ${ }^{2}$ \\ ${ }^{1}$ Department of Electrical Engineering, Iran University of Science and Technology, Tehran, Iran 16846 \\ ${ }^{2}$ Department of Computer Science, Ryerson University, Toronto ON, Canada M5B 2K3
}

\begin{abstract}
In this paper we discuss about our new clustering algorithm (ALEC) by modeling the $\mathrm{CH}$ and non $\mathrm{CH}$ nodes during set-up and steady-state phases of the algorithm. We investigate the effects of event sensing reliability and number of clusters on the network lifetime. We also analyze the power consumption and delay overheads of the clustering algorithm. According to the results, energy consumption overhead and delay overhead of ALEC algorithm in worst cases are very low (overhead of power consumption is about $0.06 \%$ and overhead of delay is about $0.001 \%$.). The results also show that longer network lifetime can be achieved by both lower values of number of clusters and sensing reliability.
\end{abstract}

Index Terms-Adaptive Low-Energy Clustering, cluster-head election, clustering overhead, sensing reliability, IEEE 802.15.4, network lifetime, wireless sensor networks.

\section{INTRODUCTION}

Recently rapid development of Low Rate-Wireless Personal Area Network (LR-WPAN) technology in the field of Wireless Sensor Network (WSN) makes ZigBee technology one of the most popular technological inventions. ZigBee technology will act as an indispensable role in various fields such as industry, medical care, and intelligent home, by its unique features, including low power consumption, low rate, low cost and high efficiency, and high reliability [15]. Replacing batteries of nodes is infeasible because of the large number of nodes and possibly harsh environment in which they are deployed; therefore, the most important problem in these networks is to perform its operations in an efficient manner to prolong their lifetimes. One of the most famous methods for reducing energy consumption in network is clustering. According to clustering, sensor nodes in a network divided into groups according to specific requirements or metrics. As shown in Fig. 1 , in each group or cluster a sensor node is chosen as a leader referred to as Cluster-Head $(\mathrm{CH})$. A CH node is responsible for conveying any information gathered by nodes in its cluster to BS. Clustering has some benefits as follows: a) reducing the transmission overhead for the update of routing tables after topological changes. b) Clustering allows intra-cluster and inter-cluster routing which reduces the number of nodes taking part in a long distance communication, thus allowing significant energy saving in addition to smaller dissemination latency. c) Dividing network into clusters reduces number of contending nodes and consequently lowers collisions made by nodes during medium access.

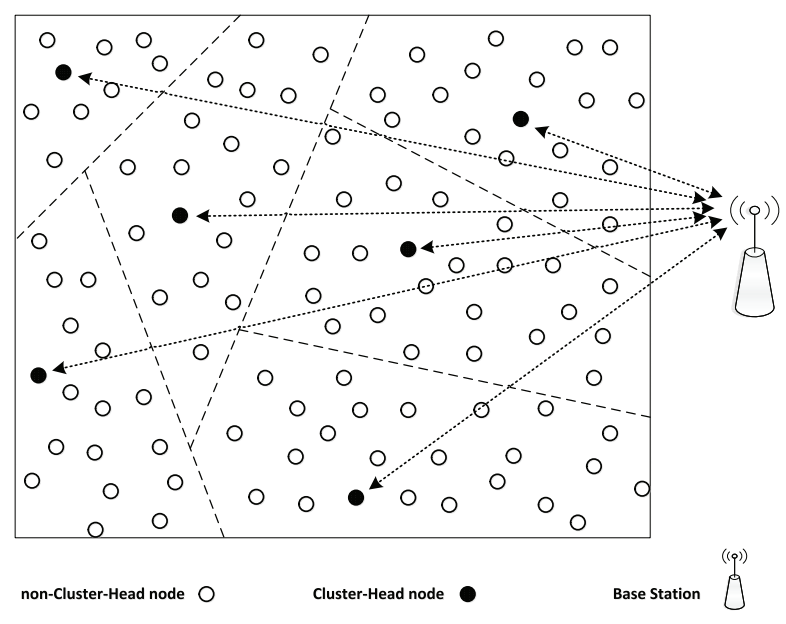

Fig. 1. Topology of the network for round $i$.

Since added responsibility results in a higher rate of depleting energy at $\mathrm{CHs}$, an effective solution for prohibiting $\mathrm{CH}$ nodes from early dying is to rotate cluster-head roles among nodes. However, cluster-head election and forming new clusters require some message exchanges between nodes. In the other words, periodically changing cluster-heads requires consuming some energy and spending some time for establishment of new clusters on the network. To the best of our knowledge there is no analysis of integrated communication and cluster-head changing functions in wireless sensor network. Furthermore, we believe this paper serves as a useful starting point for the researchers who are interested in conducting research in evaluating clustering algorithms and their overhead on the network.

In this paper a detailed model of traffic caused by sensing and by clustering algorithm in the network is presented. We also model the energy consumption of message exchanges associated with ALEC algorithm. We assume that individual sensor nodes are battery operated and their transceivers are modeled after the 2.4 GHz IEEE 802.15.4 / ZigBee-ready RF Transceiver [1]. The paper is organized as follows. Section III gives a brief overview of operation of 802.15.4-compliant networks with star topology, followed by a review of power consumption parameters. An overview of ALEC algorithm is presented in section IV. Sections V and VI present deriva- 
tion of analytical model for energy consumption of ALEC algorithm. Section VII presents numerical performance results. Finally, Section VIII concludes the paper.

\section{RELATED WORK}

A clustering algorithm composed of rounds each of which composed of two major phases: set-up phase and steady-state phase. Since sensor nodes have limited capabilities in terms of communication range, energy resource and processing power, the clustering algorithms should be compatible with these limitations in either set-up or steady-state phases. There are two major clustering approaches: distributed and centralized. In distributed approaches, the decisions for next $\mathrm{CH}$ elections are individually made by ordinary nodes or by $\mathrm{CH}$ nodes while in centralized algorithms, there is a central node in the network that is responsible of electing new $\mathrm{CH}$ nodes.

Distributed approaches usually consist of probabilistic methods in which selections of $\mathrm{CH}$ nodes are based on evaluation of expressions. These expressions usually composed of some parameters, e.g. round number, number of $\mathrm{CHs}$ or amount of energy. One of the most popular probabilistic schemes is Low-Energy Adaptive Clustering Hierarchy (LEACH) [6]. In $\mathrm{LEACH}$, the randomization is used to distribute the energy consumption among all nodes in the network. There are some other algorithms trying to improve threshold value of LEACH algorithm using some other parameters like energy consumption, amount of traffic, number of neighbors and density of sensor nodes. Authors in [4] considered the ratio of the current energy level to the initial energy level of the node as a coefficient of the threshold value in LEACH algorithm. The nodes with higher level of remaining energy have more chance to be $\mathrm{CHs}$ in following rounds.

Selection of the nodes from denser areas leads to conserving more energy [14]. The algorithms in [2] and [3] consider the factor of number of neighbor nodes to select $\mathrm{CHs}$ and lowers the intra-cluster communication. However, detection of neighboring nodes imposes overhead to the network and delays clustering formation time.

Although distributed algorithms have some advantages from energy consumption overhead and delay overhead viewpoints, but lacking general knowledge of the entire network by a single node cannot result in a good efficiency in clustering of the network. Having more energy resources and more processing power makes BS a good choice for shifting the burden of $\mathrm{CH}$ selection and cluster formation phases. In centralized clustering approaches, selection of future $\mathrm{CHs}$ is decided by a node (usually BS). However, these approaches require the periodic communication with $\mathrm{BS}$ by sensor nodes to update the necessary information about current situation of the network. These approaches impose more energy and more delay overhead on the network.

There are some centralized methods based on LEACH scheme. In [7] the authors proposed LEACH-Centralized (LEACH-C) to improve placement and number of $\mathrm{CHs}$ in LEACH algorithm. The authors in [5] proposed a hybrid method in such a way that the selection of $\mathrm{CH}$ s is distributed while controlling the number of $\mathrm{CHs}$ is centralized.

Although re-clustering is proposed to increase network lifetime by evenly distributing the heavy load of $\mathrm{CH}$ roles among sensor nodes, re-clustering itself is an energy consuming procedure, which imposes extra transmission of control messages on the network [14]. Re-clustering also delays the real-time transmission of data during cluster set-up phases. In the following, the authors propose some solutions to reduce re-clustering overhead.

The paper, [10], proposes an idea that most nodes around the current $\mathrm{CH}$ have a high chance of belonging to the same cluster. Therefore, other added nodes should only be considered during exchange control packets. In another method [8], the authors considered a threshold for triggering re-clustering phase. In other words, only those $\mathrm{CHs}$ which have energy level below the threshold will participate in $\mathrm{CH}$ selection and other $\mathrm{CHs}$ will remain as $\mathrm{CHs}$ during following round.

\section{802.15.4 OPERATION AND POWER MANAGEMENT}

In the network, all nodes operate in beacon enabled, slotted CSMA-CA mode under the control of their respective cluster coordinators. In each cluster, the channel time is divided into superframes bounded by beacons transmitted by coordinators [9]. All communications in clusters take place during active portions of their superframes $S D$ s. The duration of the superframe is determined by $S O$ variable according to following relation: $S D=16 \times 3 \times 2^{S O}$ unit backoff periods. If clusters operate in the ISM band at $2.4 \mathrm{GHz}$, the duration of unit backoff period is $0.32 \times 10^{-3} s$ which results in maximum data rate of $0.25 \mathrm{Mbps}$. The time interval between successive beacons is $B I=16 \times 3 \times 2^{B O}$. Both $B O$ and $S O$ can take values according to following relation [13]: $0 \leq S O \leq B O \leq 15$. Data transfers in the uplink direction use CSMA-CA algorithm aligned to the backoff period boundary. Data transfers in the downlink direction use a more complex protocol in such a way that coordinator announces the presence of a packet, which must be explicitly requested by the target node before being actually sent [11].

Power management consists of adjusting the frequency and ratio of active and inactive periods of sensor nodes [17], [18]. Coordinator periodically broadcasts required event sensing reliability (number of packets per second needed for reliable event detection) and number of nodes which are alive. Every node should compute average duration of sleep between transmissions Based on the above information [13]. Energy consumptions for a node with a $2.4 \mathrm{GHz}$ IEEE 802.15.4 / ZigBee-ready RF Transceiver [1] operating under typical conditions in the ISM band during a backoff period, i.e. 10 bytes, are $\omega_{s}=18.2 n \mathrm{~J}, \omega_{r}=17.9 \mu \mathrm{J}$ and $\omega_{t}=15.8 \mu \mathrm{J}$, for sleep, receiving and transmitting (at $0 \mathrm{dBm})$, respectively.

\section{Adaptive Low-Energy Clustering Algorithm}

Here, we explain our new clustering algorithm (ALEC) in more details. As shown in Fig. 2, at the beginning of round $r$, a random number is chosen uniformly between 0 and 1 by 


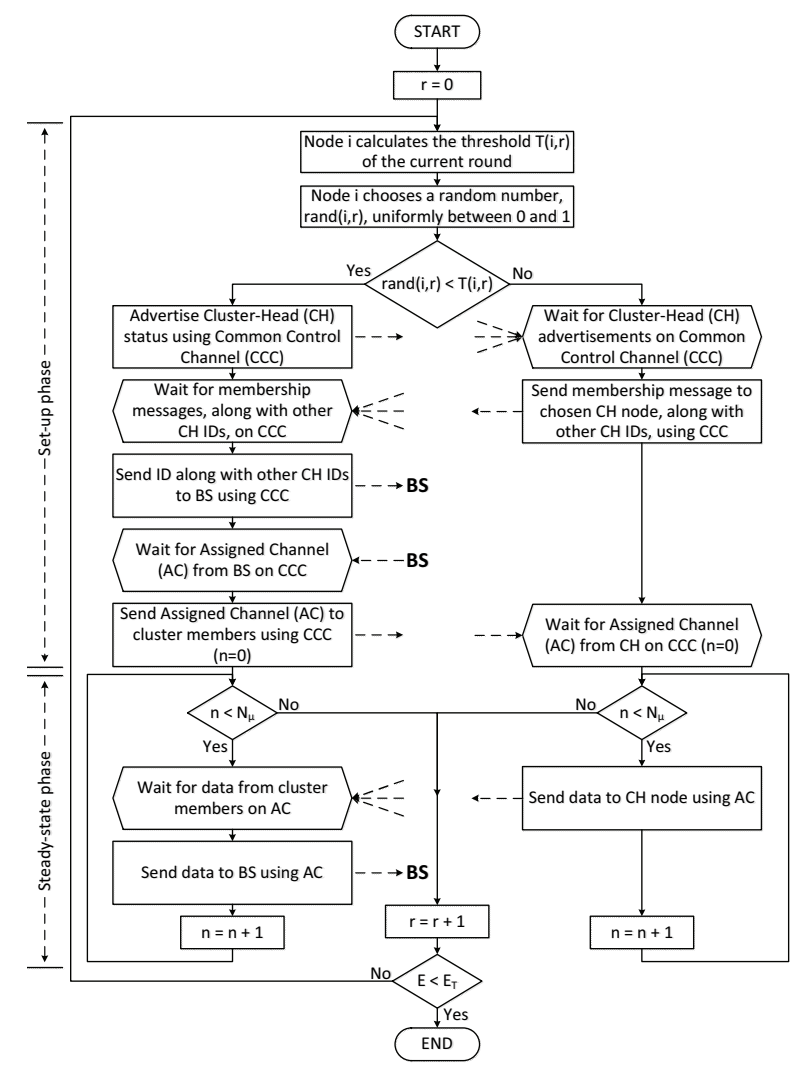

Fig. 2. flowchart of ALEC algorithm.

node $i$, and compared with a threshold $\mathrm{T}(\mathrm{i}, \mathrm{r})$. If the random number is less than the threshold, the node becomes a clusterhead. The threshold is set as:

$$
T(i, r)= \begin{cases}\frac{N_{c}}{N-N_{c} \times\left(r \bmod \frac{N}{N_{c}}\right)}, & i \in G \\ 0, & \text { otherwise }\end{cases}
$$

Where $N_{c}$ is desired number of cluster-heads, $N$ is number of nodes and $G$ is the set of nodes that have not been clusterheads in $r \bmod \frac{N}{N_{c}}$ previous rounds.

If all clusters use the same frequency channel during steadystate phase, some of nodes in each cluster, especially those near the borders, can hear signals related to adjacent clusters. This means that for each cluster, there may be some other interfering signals related to its neighbor clusters. Therefore, there may be some nodes in each cluster that cannot communicate with their own CHs. Since 802.15.4 standard uses 16 channels in the ISM band, interference between clusters can be resolved by proper channel assignment to each cluster. In the other words, all $\mathrm{CHs}$ have to receive proper frequency channel from BS in set-up phases. Channel assignment can be carried out by BS using frequency planning concept from cellular networks [16] with channel reuse factors of $\frac{1}{7}$ or $\frac{1}{12}$. According to previous discussion, each set-up phase can be divided into five sub-phases as follows:

- Advertisement: After electing as a $\mathrm{CH}$, new $\mathrm{CH}$ node starts broadcasting its status to other nodes.

- Membership: Each non $\mathrm{CH}$ node determines to which cluster it wants to belong by choosing the $\mathrm{CH}$ that requires the minimum communication energy. The non $\mathrm{CH}$ node transmits a join-request message composing of all IDs related to other $\mathrm{CHs}$ that it could hear during advertisement phase.

- Channel Request: All CHs have to inform BS about their neighbor clusters. Therefore, all $\mathrm{CHs}$ have to send their IDs along with other IDs they received in membership phase to BS.

- Channel Assignment: During this sub-phase, appropriate frequency channels are sent by BS to all $\mathrm{CH}$ nodes.

- Channel Declaration: Each CH node informs all its members about new assigned channel.

For communication with each other during set-up phases, all nodes can only use an initially dedicated frequency channel known as Common Control Channel (CCC). Each steady-state phase is composed of a number of $\left(N_{\mu}\right)$ packet transmissions. We denote this parameter $\left(N_{\mu}\right)$ as clustering period. Clusterhead nodes are awake during steady-state phases. However, non cluster-head nodes sleep between transmissions.

\section{Modeling of Clustering}

In order to model performance of the clustering, we integrate power managed sensing function with clustering algorithm. Both uplink and downlink packet transmissions use slotted CSMA-CA determined by the standard [9]. A general Markov sub-chain for a single CSMA-CA transmission is shown in Fig. 3. Delay line from Fig. 3 models the requirement from the standard that a transmission has to be delayed to the beginning of the next superframe. This probability is denoted as $P_{d}=\frac{\bar{D}_{d}}{S D}$ where $\bar{D}_{d}=2+\bar{G}_{p}+1+\bar{G}_{a}$ denotes average packet transmission time including two clear channel assessments, transmission time $\bar{G}_{p}$, waiting time for the acknowledgement and acknowledgement transmission time $\bar{G}_{a}$. The block labeled $T_{r}$ denotes $\bar{D}_{d}$ linearly connected backoff periods needed for actual transmission.

Synchronization time, i.e. the duration from the moment when node wakes up till the next beacon, is uniformly distributed between 0 and $B I-1$ backoff periods. Its Probability Generating Function (PGF) is $D(z)=\frac{1-z^{B I}}{B I(1-z)}$.

Assuming that PER represents the Packet Error Rate, the probability that the packet will not be affected by noise is $\delta=1-P E R=(1-B E R)^{\bar{G}_{p}+\bar{G}_{a}}$ where BER represents the Bit Error Rate of the medium.

As shown in Fig. 3, input probability to a transmission block is $\tau_{0} \gamma \delta$ where $\tau_{0}=\sum_{i=0}^{m} x_{i, 0,0}$ is medium access probability. Considering that medium access control layer is reliable, i.e. it will repeat transmission until the packet is acknowledged, the probability of finishing the first backoff phase in transmission block is equal to $x_{0,2,0}=\tau_{0} \gamma \delta+\tau_{0}(1-\gamma \delta)=\tau_{0}$. Using transition probabilities indicated in Fig. 3, we adopt the method in [12] and [11] and derive the relationships between different states and solve the Markov chain. Total access probability $(\tau)$ by a node in each round is equal to 


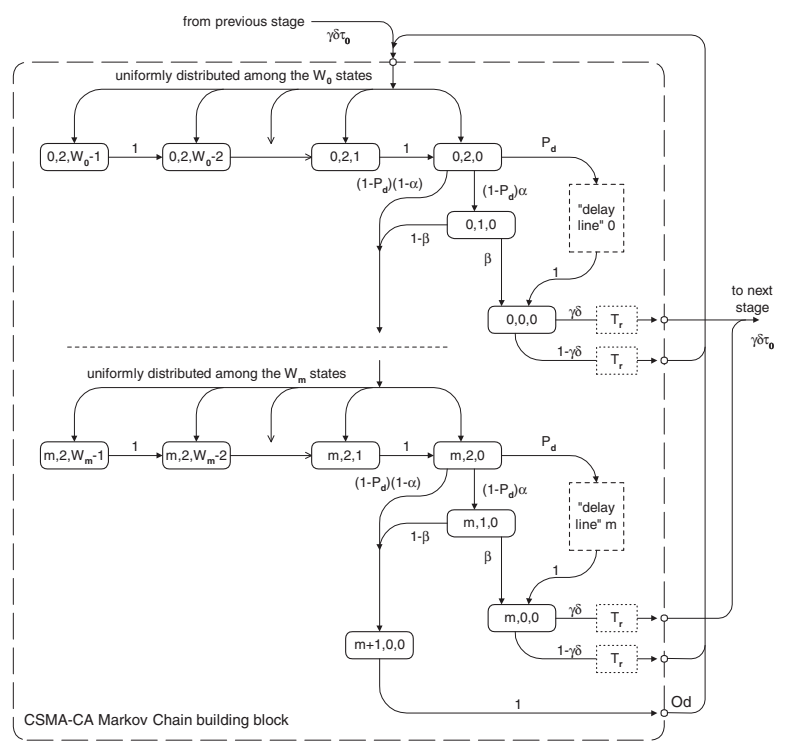

Fig. 3. Markov sub-chain for one CSMA-CA transmission [11].

the sum of access probabilities in a set-up phase and in a steady-state phase. Since number of accesses during set-up and steady-state phases are respectively $6 \tau_{0}$ and $N_{\mu} \tau_{0}$, we have $\tau=\left(6+N_{\mu}\right) \tau_{0}$, where $N_{\mu}$ is number of packets transmitted during a steady-state phase of a round.

\section{A. Modeling of inactive time}

In order to model inactive time, we assume that sleep period is geometrically distributed with parameter $P_{\text {sleep }}$. The PGF for one sleep period is $V(z)=\sum_{k=1}^{\infty}\left(1-P_{\text {sleep }}\right) P_{\text {sleep }}^{k-1} z^{k}=$ $\frac{\left(1-P_{\text {sleep }}\right) z}{1-P_{\text {sleep }} z}$. The average value of one sleep period is $\bar{V}=$ $\left.\frac{d}{d z} V(z)\right|_{z=1}=\frac{1}{1-P_{\text {sleep }}}$.

We assume arriving packets to each node follow the Poisson process with the rate $\lambda$. Therefore, the PGF of the number of packets arrive to the buffer during the a period of a node is equal to $F(z)=V^{*}(\lambda-\lambda z)$ where $V^{*}(s)=\frac{\left(1-P_{\text {sleep }}\right) e^{-s}}{1-P_{\text {sleep }} e^{-s}}$ denotes the Laplace-Stieltjes transform (LST) of the sleep period which can be obtained by substituting the variable $\mathrm{Z}$ with $e^{-s}$.

We also consider buffer of a node as $\mathrm{M} / \mathrm{G} / 1 / \mathrm{K}$ queuing model with vacations. After waking up, if there are any packets in the node's buffer, the node transmits only one packet and goes to sleep again and if there is no packet in the buffer, the node starts immediately another sleep period. This policy is known as 1-limited service policy [19]. We are able to derive approximate value of successive sleep periods for small buffer sizes of 1-2 packets which is a reasonable assumption for sensor networks. Since the packet service period is much smaller than the sleep period, new sleep will be started only if there were zero packet arrivals during the current sleep period, i.e., with probability $F(0)=V^{*}(\lambda)$. Therefore, the PGF of duration of consecutive sleep periods is $I(z)=\frac{\left(1-V^{*}(\lambda)\right) V(z)}{1-V^{*}(\lambda) V(z)}$ with the average value of $\bar{I}=\frac{1}{\left(1-P_{\text {sleep }}\right)\left(1-V^{*}(\lambda)\right)}$. In the following sections we determine relation between $\mathrm{R}$ and $\bar{I}$.

\section{B. Success probabilities}

Here we want to determine success probabilities; i.e. the probabilities that the medium is idle on first $(\alpha)$ and second $\operatorname{CCA}(\beta)$ and also the probability that the transmission is successful $(\gamma)$.

We focus on a single target node and model aggregate packet arrival rates of the remaining $\left(n_{c}-1\right)$ nodes as background traffic. This approximation is possible when event sensing reliability per cluster $\left(\frac{R}{N_{c}}\right)$ is not high, i.e., when the cluster operates below the saturation regime. We estimate the arrival rate for background traffic as: $\lambda_{c}=\left(n_{c}-1\right) \tau S D / 8$.

The first CCA may fail because a packet transmission from another node is in progress; this particular backoff period may be at any position with respect to that packet. Thus $\alpha=\frac{1}{8} \sum_{i=1}^{7} e^{-i \lambda_{c}}$. Note that the first medium access will happen within the first 8 backoff periods of the superframe. The second CCA, however, will fail only if some other node has just started its transmission. Thus $\beta=e^{-\lambda_{c}}$. The probability of success of a transmission attempt is $\gamma=\beta^{\bar{D}_{d}}$.

Access probability for $\mathrm{CHs}$ (bridges) can be modeled as $\tau_{b r i}=n_{c} \tau$. The success probability for bridge transmissions depends on all other bridges, hence $\gamma_{b r i}=\left(1-\tau_{b r i}\right)^{\bar{D}_{d}\left(N_{c}-1\right)}$.

\section{NODE LIFETIME}

If we assume that the length of a packet is $k$ backoff periods, then the PGF of packet length is $G_{p}(z)=z^{k}$. The PGF of the time interval between the data and subsequent ACK packet is $t_{a c k}(z)=z^{2}$. We also denote the PGF for packet transmission time and receipt of acknowledgement as $T_{d}(z)=G_{p}(z) t_{a c k}(z) G_{a}(z)$. We can also determine the PGF for the time needed for one complete transmission attempt, including backoffs [11], as: $\mathcal{A}(z)=$ $\frac{\sum_{i=0}^{m}\left(\prod_{j=0}^{i} B_{j}(z)\right)(1-\alpha \beta)^{i} z^{2(i+1)}\left(\alpha \beta T_{d}(z)\right)}{\alpha \beta \sum_{i=0}^{m}(1-\alpha \beta)^{i}}$. The LST for the energy consumption during $j$-th backoff time prior to transmission is $E_{B_{j}}^{*}(s)=\frac{e^{-s \omega_{r} W_{j}}-1}{W_{j}\left(e^{-s \omega_{r}}-1\right)}$.

The LSTs for energy consumption during pure packet transmission time, two CCAs and wait and reception of the acknowledgment are respectively $e^{-s k \omega_{t}}, e^{-s 2 \omega_{r}}$ and $e^{-s 3 \omega_{r}}$ [13]. The LST of energy consumption for receiving beacon containing information about the number of alive nodes and requested event sensing reliability is $e^{-s 3 \omega_{r}}$. Then, the LST for energy consumption during transmission time of the data packet and reception of acknowledgement will be denoted with $T_{d}^{*}(s)=e^{-s k \omega_{t}} e^{-s 2 \omega_{r}} e^{-s \omega_{r}}$. The LST for energy consumption for one transmission attempt becomes $\mathcal{E}_{\mathcal{A}}^{*}(s)=\frac{\sum_{i=0}^{m}\left(\prod_{j=0}^{i} E_{B_{j}}^{*}(s)\right)(1-\alpha \beta)^{i} e^{-s 2 \omega_{r}(i+1)} \alpha \beta T_{d}^{*}(s)}{\alpha \beta \sum_{i=0}^{m}(1-\alpha \beta)^{i}}$. By taking packet collisions into account [12], the PGF of probability distribution of the packet service time becomes $T(z)=\sum_{k=0}^{\infty}(\mathcal{A}(z)(1-\gamma \delta))^{k} \mathcal{A}(z) \gamma \delta=\frac{\gamma \delta \mathcal{A}(z)}{1-\mathcal{A}(z)+\gamma \delta \mathcal{A}(z)}$ and the LST for the energy spent on a packet service time is $E_{T}^{*}(s)=\frac{\gamma \delta \mathcal{E}_{\mathcal{A}}^{*}(s)}{1-\mathcal{E}_{\mathcal{A}}^{*}(s)+\gamma \delta \mathcal{E}_{\mathcal{A}}^{*}(s)}$. Average value of energy consumed for packet service is [12]: $\bar{E}_{T}=-\left.\frac{d}{d s} E_{T}^{*}(s)\right|_{s=0}$. 
$\mathrm{CH}$ nodes send packets during advertisement, channel request, uplink request for channel assignment and channel declaration sub-phases of a set-up phase. They receive packets during membership and downlink data for channel assignment sub-phases of a set-up phase. There are also four synchronization times during a set-up phase: synchronization for advertisement, membership, channel request and uplink request for channel assignment. Non $\mathrm{CH}$ nodes only send packets during membership sub-phase. They are in receiving mode during other sub-phases of a set-up phase. According to above discussion, average energy consumption during one set-up phase for a CH node is $\bar{E}_{s u, C H}=4 \bar{E}_{T}+2 \bar{T} \omega_{r}+4(\bar{D}+3) \omega_{r}$, and for a non $\mathrm{CH}$ node is $\bar{E}_{s u, n C H}=\bar{E}_{T}+5 \bar{T} \omega_{r}+4(\bar{D}+3) \omega_{r}$. If we assume $\omega_{r} \approx \omega_{t}$, then $\bar{E}_{s u, C H} \approx \bar{E}_{s u, n C H}$.

Each steady-state phase is composed of a number $\left(N_{\mu}\right)$ of microcycles which is composed of three steps: sleep, beacon synchronization and data transmission (CSMA uplink). However, all $\mathrm{CH}$ nodes are awake during a round. Average energy consumption for $\mathrm{CH}$ nodes during a microcycle is $\bar{E}_{m i, C H}=\bar{D} \omega_{r}+3 \omega_{r}+\bar{I} \omega_{r}+\bar{T} \omega_{r}$, and for non $\mathrm{CH}$ nodes is $\bar{E}_{m i, n C H}=\bar{D} \omega_{r}+3 \omega_{r}+\bar{I} \omega_{s}+\bar{T} \omega_{r}$. Average energy consumption during one round for $\mathrm{CH}$ nodes is $\bar{E}_{r d, C H}=\bar{E}_{s u, C H}+N_{\mu} \bar{E}_{m i, C H}$, and for non $\mathrm{CH}$ nodes is $\bar{E}_{r d, n C H}=\bar{E}_{s u, n C H}+N_{\mu} \bar{E}_{m i, n C H}$.

A macrocycle is composed of $n_{c}$ rounds. Each node has to be $\mathrm{CH}$ only for one round during a macrocycle. Therefore, the energy consumed during one macrocycle is $\bar{E}_{m a}=\bar{E}_{r d, C H}+$ $\left(n_{c}-1\right) \bar{E}_{r d, n C H}$. If the battery budget is $E_{b a t}$ Joules, the average number of macrocycles during lifetime of a node is $\frac{E_{b a t}}{\bar{E}_{m a}}$. Therefore, lifetime of the network is $\bar{L}=\bar{T}_{m a} \times \frac{E_{b a t}}{\bar{E}_{m a}}$ where $\bar{T}_{m a}$ is duration of a macrocycle.

\section{PERFormance EVALUATION}

Here we present numerical results obtained by solving the system of equations presented in sections V and VI and obtain some of system parameters e.g. $\tau_{0}, \tau, \alpha, \beta, \gamma$ and $P_{\text {sleep }}$. There are 400 nodes in the network. We assumed that each node is powered with two AA batteries which supply voltage between 2.1 and $3.6 \mathrm{~V}$ with total energy $E_{b a t}=2 \times 5130 \mathrm{~J}=10260 \mathrm{~J}$. We have assumed that $B E R=10^{-4}$ and network operates in the ISM band at $2.4 \mathrm{GHz}$, with raw data rate $\frac{1}{4}$ Mbps. Superframe size (SD) and beacon interval (BI) are respectively adjusted at 48 and 96 backoff periods; i.e. $S O=0$ and $B O=$ 1.

According to relations of $\delta$ and $\gamma$, increasing value of $\bar{G}_{p}$ results in decreasing $\delta$ and $\gamma$ which means more retransmissions and consequently consuming more energy. In order to consider the worst case, the longest packet size according to IEEE 802.15.4 standard is considered ( $\bar{G}_{p}=12$ backoff periods). We also assume that each node has a buffer size of 2 packets.

We want to investigate the performance of the ALEC algorithm according to event sensing reliability $(R)$, i.e. number of packets per second needed for reliable event detection, and number of clusters $\left(N_{c}\right) . R$ and $N_{c}$ are set by application and cannot be changed by network administrator. In Fig. 4 number of microcycles is constant at $500\left(N_{\mu}=500\right)$. Number of clusters is variable in the range 8 to 24 in steps of 4 . Hence, there are $n_{c}$ nodes in each cluster $\left(n_{c}=\frac{N}{N_{c}}\right)$ which is variable in the range 16 to 50 in steps of 8 . Event sensing reliability $(\mathrm{R})$ is variable in the range 10 to 50 packets per second in steps of 10 . This means that number of packets should be sent to BS by each node $\left(r=\frac{R}{N}\right)$ is variable in the range 0.025 to 0.125 packets per second in steps of 0.025 . Therefore, we can determine average duration of a microcycle as $\bar{T}_{m i}=\frac{1}{r}=\bar{I}+\bar{D}+3+\bar{T}$.

According to figures 4(a) and 4(b), we can compare energy consumptions of $\mathrm{CH}$ and non $\mathrm{CH}$ nodes. As can be seen in these figures, energy consumption will be increased if we decrease sensing reliability. As mentioned before, during setup phases no data can be transmitted to BS. This means that set-up phases create some delay in network operation. The ratio of the total delay to the lifetime of the network, i.e. overhead of delay, is shown in Fig. 4(c). As can be seen in the figure, in worst cases (lower values of $N_{c}$ and higher values of $\mathrm{R}$ ), overhead of delay is around $0.001 \%$ which is very low. In Fig. 4(d), the rate of energy consumption by the network is shown. As can be seen in the figure energy consumption of the network will be reduced if higher values of $N_{c}$ are adopted. We also can see that increasing $R$ results in increasing energy consumption.

Energy consumption during set-up phases is the overhead of energy consumption of the clustering algorithm because sensor nodes contribute the energy to the network to establish new clusters. The overhead of energy consumption is shown in Fig. 4(e) as the ratio of total energy consumed by a node during set-up phases to the total energy consumption of a node. Reducing overhead of power can be done by adopting higher values of $N_{c}$ or lower values of R. As can be seen in the figure, in worst cases (lower values of $N_{c}$ and higher values of $\mathrm{R}$ ), overhead of power consumption is around $0.06 \%$ which is very low. The lifetime of the network is shown in Fig. 4(f). Since lower values of $R$ result in longer inactive periods between packet transmissions, higher values of lifetime can be reached by adopting lower values of R. Each macrocycle is composed of $n_{c}$ rounds. Since lower values of $N_{c}$ result in longer macrocycles, lifetime of the network can also be extended by choosing lower values of $N_{c}$.

\section{CONCLUSION}

In this paper, we consider our new Adaptive Low-Energy Clustering (ALEC) algorithm operating with IEEE 802.15.4 beacon enabled mode. We evaluate the impact of event sensing reliability and number of clusters on the network lifetime. According to ALEC, rotating the role of $\mathrm{CHs}$ and forming new clusters require message exchanges between nodes and impose power consumption overhead on the network. The results show that energy consumption overhead and delay overhead of ALEC algorithm in worst cases are very low (overhead of power consumption is about $0.06 \%$ and overhead of delay is about $0.001 \%$.). According to the results, longer network lifetimes can be achieved by choosing either lower 

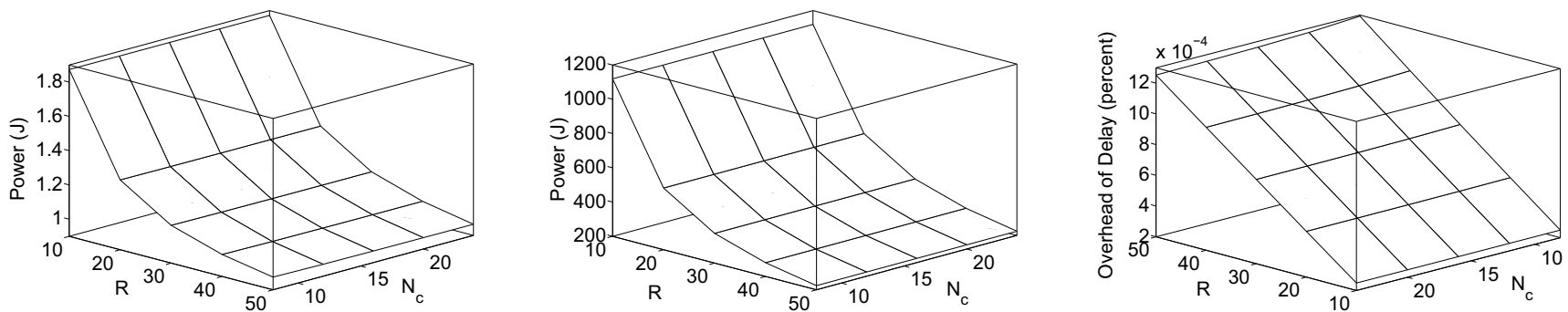

(a) Power consumption (in Joules) during a steady- (b) Power consumption (in Joules) of a CH node (c) Overhead of delay of the clustering algorithm state phase of a non $\mathrm{CH}$ node according to different during one round according to different values of (in percent) according to different values of sensing values of sensing reliability $(R)$ and number of sensing reliability $(R)$ and number of clusters $\left(N_{c}\right)$. reliability $(R)$ and number of clusters $\left(N_{c}\right)$. clusters $\left(N_{c}\right)$
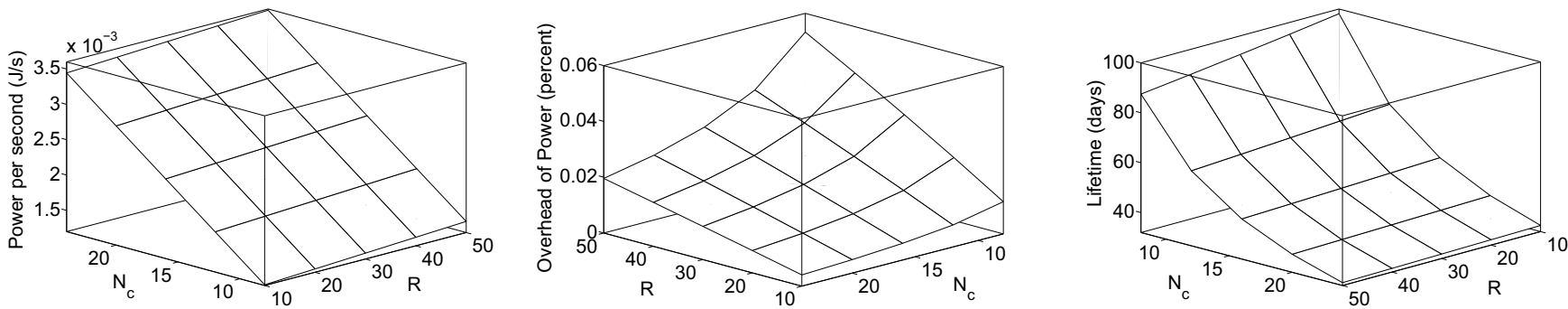

(d) Power consumption per second of a node ac- (e) Overhead of power consumption of the clus- (f) Lifetime of a node (in days) according to differcording to different values of sensing reliability $(R)$ tering algorithm (in percent) according to different ent values of sensing reliability $(R)$ and number of and number of clusters $\left(N_{c}\right)$. values of sensing reliability $(R)$ and number of clusters $\left(N_{c}\right)$ clusters $\left(N_{c}\right)$.

Fig. 4. Lifetime and power consumptions of ALEC algorithm according to different values of sensing reliability $(R)$ and number of clusters $\left(N_{c}\right)$.

values of event sensing reliability or lower values of number of clusters. Our method can also be easily scaled to any other clustering algorithms.

\section{REFERENCES}

[1] Chipcon CC2420 Datasheet: 2.4 GHz IEEE 802.15.4 / ZigBee-Ready RF Transceiver (Rev. B), 2012.

[2] X. Bian, X. Liu, and H. Cho. Study on a cluster-chain routing protocol in wireless sensor networks. In Communications and Networking in China, 2008. ChinaCom 2008. Third International Conference on, pages 964 968, Aug. 2008

[3] R.-S. Chang and C.-J. Kuo. An energy efficient routing mechanism for wireless sensor networks. In Advanced Information Networking and Applications, 2006. AINA 2006. 20th International Conference on, volume 2, page 5 pp., April 2006.

[4] M. Handy, M. Haase, and D. Timmermann. Low energy adaptive clustering hierarchy with deterministic cluster-head selection. In Mobile and Wireless Communications Network, 2002. 4th International Workshop on, pages 368-372, 2002.

[5] P. Hao, W. Qiu, and R. Evans. An Improved Cluster-head Selection approach in wireless sensor networks. In Intelligent Sensors, Sensor Networks and Information Processing (ISSNIP), 2009 5th International Conference on, pages 79-84, Dec. 2009.

[6] W. Heinzelman, A. Chandrakasan, and H. Balakrishnan. Energy-efficient communication protocol for wireless microsensor networks. In System Sciences, 2000. Proceedings of the 33rd Annual Hawaii International Conference on, page $10 \mathrm{pp}$. vol.2, Jan. 2000.

[7] W. Heinzelman, A. Chandrakasan, and H. Balakrishnan. An applicationspecific protocol architecture for wireless microsensor networks. Wireless Communications, IEEE Transactions on, 1(4):660-670, Oct. 2002.

[8] J. Hong, J. Kook, S. Lee, D. Kwon, and S. Yi. T-LEACH: The method of threshold-based cluster head replacement for wireless sensor networks. Information Systems Frontiers, 11(5):513-521, Nov. 2009.
[9] IEEE. Wireless MAC and PHY specifications for low rate WPAN. IEEE Std 802.15.4-2006 (Revision of IEEE Std 802.15.4-2003), IEEE, New York, NY, 2006

[10] J. Kim, J. Lee, and K. Rim. 3DE: selective cluster head selection scheme for energy efficiency in wireless sensor networks. In Proceedings of the 2nd International Conference on PErvasive Technologies Related to Assistive Environments, PETRA '09, pages 33:1-33:7, New York, NY, USA, 2009. ACM.

[11] J. Mišić. Traffic and energy consumption of an IEEE 802.15.4 network in the presence of authenticated, ECC Diffie-Hellman ephemeral key exchange. Computer Networks, 52(11):2227-2236, 2008.

[12] J. Mišić. Cost of secure sensing in IEEE 802.15.4 networks. Wireles. Communications, IEEE Transactions on, 8(5):2494-2504, may 2009.

[13] J. Mišić and V. Mišić. Wireless Personal Area Networks Performance, Interconnections and Security with IEEE 802.15.4. Wiley Publishing, Inc., first edition, 2008.

[14] S. Naeimi, H. Ghafghazi, C.-O. Chow, and H. Ishii. A Survey on the Taxonomy of Cluster-Based Routing Protocols for Homogeneous Wireless Sensor Networks. Sensors, 12(6):7350-7409, 2012.

[15] Z. Qian, C. Cheng, X. Zhang, Y. Wang, and I. Stojmenovic. Cluster Label-Based Routing Strategy for Saving Energy in ZigBee Mesh Network. In Recent Advances in Computer Science and Information Engineering, volume 127 of Lecture Notes in Electrical Engineering, pages 339-345. Springer Berlin Heidelberg, 2012.

[16] T. Rappaport. Wireless communications: Principles \& Paractice. Prentice Hall, Upper Saddle River, NJ 07458, 1996.

[17] Y. Sankarasubramaniam, Ö. B. Akan, and I. F. Akyildiz. ESRT: eventto-sink reliable transport in wireless sensor networks. In Proc. 4th ACM MobiHoc, pages 177-188, Annapolis, MD, June 2003.

[18] I. Stojmenović, editor. Handbook of Sensor Networks: Algorithms and Architectures. John Wiley \& Sons, 2005.

[19] H. Takagi. Queueing Analysis, volume 1: Vacation and Priority Systems. North-Holland, Amsterdam, The Netherlands, 1991. 